\title{
Gestión del conocimiento en intranets corporativas: desarrollo de un modelo de diseño
}

\author{
José Luis González Ugarte
}

Universitat Rovira i Virgili (España)

"Intranets are only as good as their content."

(Mansell-Lewis,1997)

\subsection{Resumen}

Se analizan las intranets como instrumento de gestión del conocimiento corporativo. Se presentan su características principales, su tipología, sus ventajas e inconvenientes, los factores de éxito en su desarrollo, sus limitaciones y los retos a los que se enfrenta su implementación eficaz. El diseño de la intranet ha de hacerse pensando más en los usuarios que en la tecnología.

Palabras clave: Intranet. Gestión de la información. Gestión del conocimiento.

\subsection{Abstract}

Intranets are considered as tools for corporative knowledge management. Their basic features, types, advantages and disadvantages, factors of success in their development, limitations and challenges that their effective implementation faces are analyzed. Intranet design must put the users in the first stage.

Keywords: Intranet. Information management. Knowledge management.

\section{Introducción}

\subsection{Definición conceptual de intranet}

Podemos definir intranet, en sentido estricto, como una red informática que utiliza los protocolos de comunicación propios de Internet, desarrollada la mayoría de veces para uso interno y exclusivo de una organización. La utilización de estos protocolos permite obtener dos grandes beneficios: Por un lado, permite establecer electrónicamente comunicación directa entre los miembros de una organización. Esta comunicación, como veremos más adelante, puede favorecer los procesos de socialización que permiten el intercambio de conocimiento tácito y explícito entre estos miembros. Por otro, permite la integración articulada y

Scire. $10: 2$ (jul.-dic. 2004) 25-39. 


\section{6}

ergonómica en el seno de una misma solución tecnológica de todos los recursos informáticos que se utilicen en la implantación de un programa de gestión del conocimiento en la organización. Además, permite utilizar en línea todos estos recursos aprovechando los estándares de Internet.

Diseñar una intranet desde la perspectiva de la gestión del conocimiento implica explotar la tecnología web en un contexto organizativo con el objeto de que los usuarios $-\mathrm{y}$, por tanto, la organización entendida como un todo- sean capaces de mejorar el uso de su conocimiento. Nuestro trabajo pretende analizar las intranets no tanto como herramienta de gestión de la información, sino como herramienta para la gestión del conocimiento, examinando las acciones que los usuarios llevan a cabo cuando interactúan con la información. Si analizamos las relaciones que se producen entre información y conocimiento, la intranet puede proporcionar un espacio que propicie la interacción siempre que el diseño aproveche las características del entorno web, esté basado en una perspectiva global, y, además, contemple a los usuarios de la organización en el papel de actores principales. Llegados a este punto, ya podemos destacar un aspecto importante: el diseño de la intranet ha de hacerse pensando más en los usuarios que en la tecnología per se.

\subsection{Características fundamentales}

Desde un punto de vista tecnológico, el entorno web aporta tres características distintivas que diferencian las intranets de otros entornos tecnológicos de información. La existencia de otro elemento diferencial entre intranet e Internet confiere a las intranets cuatro características básicas: 1) las intranets son hipertextuales - los hiperenlaces posibilitan que sean una tecnología con una orientación pull y user-driven - ; 2) las intranets están diseñadas para el trabajo en red permitiendo acceso distribuido tanto a nivel geográfico como a la hora de determinar los accesos permitidos; 3) las intranets son abiertas - al igual que Internet son una tecnología basada mayoritariamente en códigos de software y estándares abiertos-; y 4) las intranets pueden delimitarse en la organización, pues permiten restringir el acceso a sus miembros. Desde una perspectiva de gestión del conocimiento este último factor resulta importante, ya que permite que la organización pueda compartir libremente información lejos de competidores no deseados.

\subsection{Panorama actual de las intranets}

Algunos autores (Stenmark, 2002 ; Stodart, 2001) han destacado el vacío informativo existente sobre modelos de intranets corporativas que han conseguido facilitar el intercambio de conocimiento, si exceptuamos los estudios de Chase (1997). La impresión resultante es que son pocas las organizaciones que utilizan las intranets de manera efectiva para compartir conocimiento, siendo el sentimiento general que los desarrollos actuales sólo son la punta del iceberg.

Scire. $10: 2$ (jul.-dic. 2004) 25-39. 


\subsection{Tipos de intranets}

En tanto que herramientas para la gestión del conocimiento y en base al enfoque prioritario que concedan al conocimiento podemos establecer cuatro enfoques de las intranets (Alavi, 2001): a) intranets orientadas a la creación de conocimiento, b) intranets orientadas al almacenamiento y recuperación de conocimiento, c) intranets orientadas al intercambio de conocimiento; y d) intranets orientadas al uso del conocimiento.

Las intranets orientadas a la creación de conocimiento (Intranets for Knowledge Creation) son herramientas que favorecen la innovación. El conocimiento depende más de la acción de networking que de las redes en tanto que soporte tecnológico. El objetivo principal consiste en dar soporte a las redes de relación social que tienen lugar en las empresas (Mansel-Lewis, 1997).

Las intranets orientadas al almacenamiento y recuperación de conocimiento (Intranets for Knowledge Storage/Retrieval) son un enfoque que ha sido desarrollado siguiendo dos orientaciones diferentes en función de si la intranet se describe como conocimiento de base no estructurado (Tellen, 1996) o bien como medio para el libre flujo e intercambio de información (Benett, 1999) Desde la perspectiva del flujo informativo, las intranets contribuyen a la diseminación de la información y a la existencia de puntos de vista colaborativos. En este sentido, no debemos olvidar que el potencial de las intranets para gestionar el conocimiento organizativo depende fundamentalmente de una interfaz de usuario que permita a los miembros de la organización diferentes utilizaciones de la información almacenada (Standing, 2000).

Las intranets orientadas al intercambio de conocimiento (Intranets for Knowledge Sharing) permiten que el nivel de competencia de las organizaciones se fundamente en gran medida en la capacidad de sus miembros para transferir conocimiento a los demás. La existencia en la mayoría de organizaciones de silos de conocimiento, es decir, de repositorios de información vital no conectados, evidencia la necesidad de implementar sistemas que rentabilicen estos repositorios desde una perspectiva de gestión del conocimiento.

Finalmente, las intranets orientadas al uso del conocimiento (Intranets for Knowledge Use) permiten a las organizaciones interactuar con la intranet para utilizar el conocimiento de sus miembros y ver como lo utilizan. Esta tipología representaría la yuxtaposición de las orientaciones anteriores.

\section{Intranet y la gestión del conocimiento}

\subsection{La gestión del conocimiento tácito}

La mayor parte del conocimiento que poseemos es conocimiento tácito, es decir, está formado por cosas que no somos capaces de expresar, ya que sabemos Scire. $10: 2$ (jul.-dic. 2004) 25-39. 
más de lo que podemos expresar. Este tipo de conocimiento, al no estar disponible de forma explícita, se articula difícilmente, dado que no acostumbramos a ser conscientes de su disponibilidad; no sentimos la necesidad personal de hacerlo explícito ni de documentarlo, ya que es una tarea compleja y que de entrada no reporta ningún beneficio directo; y puede representar una pérdida potencial de poder y ventajas competitivas. La dificultad de convertir el conocimiento tácito en conocimiento explícito ha sido analizada por diferentes autores (Choo, 2000; Nonaka, 1995; Polanyi, 1966; Hall, 2001) destacando tanto su ambigüedad como la tensión existente entre su valor y su intangibilidad.

\subsection{La creación de contexto mediante el intercambio}

Tal como hemos visto hasta el momento, la intranet puede ser considerada como uno de los recursos tecnológicos más interesantes para fomentar el flujo y el intercambio global de conocimiento en el seno de una organización, ya que permite a sus miembros compartir su conocimiento y hacerlo más activo. La intranet puede proveer también un contexto para el diálogo, la reflexión y la perspectiva. Nonaka y Konno (Nonaka,1998) utilizaron la palabra japonesa "ba" para describir un espacio compartido de naturaleza física, virtual y mental, que puede ser entendido como un ejemplo de este entorno. Choo (Choo, 2000) también sugiere la intranet como un espacio compartido de información para el almacenaje, la comunicación y la colaboración, aunque desde una visión meramente informacional. Así pues, la gestión del conocimiento fundamentada en el uso de intranets debería caminar sobre la fina línea que existe entre las necesidades administrativas (documentación o control del conocimiento) y la creación de una infraestructura tecnológica y sociocultural apropiada que promueva la creatividad y la innovación así como el intercambio del conocimiento (Ali, 2001). Lo importante, y en este sentido el aspecto diferencial de la perspectiva que proponemos, consiste no sólo en la conversión del conocimiento tácito en conocimiento explícito (identificación, captura y conversión), sino en el diseño de soluciones tecnológicas que permitan identificar y compartir personas poseedoras de conocimiento, facilitar las interacciones sociales, visualizar y comunicar el conocimiento tácito, y explotar el conocimiento tácito a nivel organizativo. Los sistemas de recuperación de información pueden ser de gran ayuda a la hora de explotar el conocimiento tácito a nivel organizativo sin necesidad de hacerlo explícito. Los elementos tecnológicos de información - tanto por su contenido como por las acciones que conllevan - permiten superar el vacío existente entre el conocimiento tácito que se produce entre dos personas que comparten un contexto común, y facilitar, además, el intercambio de conocimiento.

Scire. $10: 2$ (jul.-dic. 2004) 25-39. 


\subsection{Intranet como escenario}

En tanto que escenario de conocimiento, Stenmark (Stenmark, 2002a) propone un análisis de las intranets desde una triple perspectiva que puede facilitar la gestión del conocimiento: a) la de la información (information); b) la de la conciencia (awareness) y, c) la de la comunicación (comunication) (figura 1).

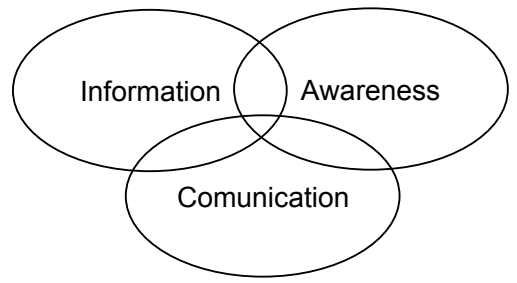

Fig 1. Perspectivas de la gestión del conocimiento (Stenmark, 2002).

La perspectiva de la información resulta la más obvia, ya que la provisión de información forma parte fundamental de la estructura: permite acceder a la información estructurada y no estructurada en forma de documentos y bases de datos. Se relaciona con el conocimiento en tanto que incrementa el acceso de los usuarios a la información y crea oportunidades para una audiencia mayor. El acceso a diferentes bloques de información valiosa resulta importante para la creación de conocimiento, ya que proporciona estímulos diversos y la redundancia necesaria (Nonaka,1998).

La perspectiva de la conciencia permite explotar no sólo el conocimiento explícito, sino también las conexiones tácitas que ayudan a los miembros de la organización a recuperar la información que de otra manera podían haber perdido. La gran cantidad de información disponible puede llegar a ser excesiva y por tanto, son necesarias herramientas que ayuden a los miembros de la organización a poderla recuperar, sobretodo cuando se añade información nueva y relevante. La red se convierte, así, en un requisito fundamental para construir la comunidad e incrementar la probabilidad de comunicación y colaboración.

La perspectiva de la comunicación permite que los miembros de la organización interpreten la información disponible mediante los diferentes canales de conversación y negociación. Promueve la reflexión, los flujos de trabajo y la coordinación de rutinas y significados para trabajar conjuntamente. La intranet debería permitir que los miembros de la organización trabajasen de manera activa basándose en la información disponible y la comunicación de sus experiencias para poder influenciarse mutuamente. 
El proceso de conversión de la información en conocimiento corporativo sigue diversas fases (Hoog, 1999): 1) captura de la información; 2) estructuración y estandarización de los contenidos - creación uniforme de documentos corporativos muy útiles para la recuperación posterior (a pesar de que a menudo no se tenga mucho cuidado en hacerlo) - ; y 3) almacenamiento y distribución de los contenidos estructurados. Las formas en que aprendemos pueden ayudarnos a entender los roles, comportamientos, herramientas y procesos que necesitamos desarrollar para ayudar a los individuos de la organización a encontrar el conocimiento disponible y promover que la organización aprenda y capture las experiencias creando un conocimiento organizativo de base con un mínimo de esfuerzo.

\section{El paradigma intranet}

Entendiendo por paradigma todas aquellas acciones y mecanismos que ayudan a definir lo que consideramos importante y la manera como nos aproximamos a los problemas y a las actividades, ¿podemos decir que las intranets representan un cambio de paradigma o la necesidad de un nuevo paradigma? $\mathrm{Si}$ todo el conocimiento fuese fácilmente codificable y almacenable, no seria necesario hablar de un nuevo paradigma para la gestión del conocimiento, ya que los sistemas de gestión de datos e información clásicos ya hubieran resuelto el tema; $\mathrm{y}$, ciertamente, muchos sistemas de información han demostrado bastante ineficacia a la hora de intentar gestionar conocimiento. El fenómeno intranet representa la lógica extensión de la naturaleza empírica e incremental del cambio que ha comportado Internet (Fishenden, 1997) y probablemente sea la manifestación más tangible del cambio de la era de la información a la era del conocimiento. El cambio de paradigma que se produce con la introducción de las intranets implica una modificación en la organización que podemos tipificar en los siete elementos clave (Eder, 2001) recogidos en la tabla I.

\begin{tabular}{|l|l|l|}
\hline 1 & Cultura & El valor se sitúa en el intercambio de conocimiento. \\
\hline 2 & Gestión & $\begin{array}{l}\text { La información y la toma de decisiones se halla distribuida como resultado de } \\
\text { una estructura orgánica flexible y adaptable. }\end{array}$ \\
\hline 3 & Objetivo & $\begin{array}{l}\text { Por su naturaleza distribuida, la gestión del conocimiento en la intranet está } \\
\text { orientada hacia la recopilación y comunicación del conocimiento. }\end{array}$ \\
\hline 4 & Coordinación & Se deriva de la necesidad de intercambio de información y conocimiento. \\
\hline 5 & Herramientas & $\begin{array}{l}\text { La arquitectura abierta y los softwares libres aseguran la flexibilidad de la infra- } \\
\text { estructura tecnológica. }\end{array}$ \\
\hline 6 & Comunicación & $\begin{array}{l}\text { Se desarrollan modelos orientados a los usuarios, los cuales reciben la informa- } \\
\text { ción en función de sus requerimientos. }\end{array}$ \\
\hline 7 & Desarrollo & $\begin{array}{l}\text { Las infraestructuras y herramientas permiten tratar la información desde un } \\
\text { punto de vista experto. }\end{array}$ \\
\hline
\end{tabular}

Tabla I. Los siete elementos clave del paradigma intranet

Scire. $10: 2$ (jul.-dic. 2004) 25-39. 
Podemos afirmar pues, que el fenómeno intranet representa un nuevo paradigma principalmente porque permite: a) comunicar y gestionar de manera diferente a como se había hecho hasta el momento; b) experimentar de manera concreta el funcionamiento y la gestión de los sistemas distribuidos (Eder, 2001); c) distribuir la toma de decisiones; d) modularizar las organizaciones; y e) identificar y reutilizar el conocimiento de los miembros de las organizaciones.

\section{Ventajas del uso de intranets}

1. De ámbito general. Si consideramos una intranet desde una perspectiva múltiple (información y comunicación) que facilite la interacción, podemos convertirla en herramienta de gestión del conocimiento que permita: a) la mejora continuada en la gestión de los procesos y de los productos, auditorias y evaluaciones; b) la compresión del espacio y del tiempo entre los usuarios; c) la gestión adecuada del capital humano y social de la empresa - requerimientos de formación, descripción de lugares de trabajo, planes de formación, currículos (Fernández de las Heras, 2000) - ; y d) la capitalización de ideas, personas e innovaciones, y la provisión de un retorno en investigación en sentido amplio.

2. Como infraestructura. Entendida como infraestructura la intranet se conforma como una solución técnicamente viable derivada de la tecnología de Internet, generalmente gratuita o barata, basada en software estándar y código abierto, reduciendo así los costes en tecnologías de la información y la comunicación.

3. Para la gestión de la información. Las tecnologías basadas en web pueden permitir la desintegración de las barreras que dificultan la libre circulación de la información, y procurar la conexión de la información en todas las direcciones, además de facilitar (Cohen, 1998): 1) la edición y publicación rápida de la información interna; 2) la mejora del aprendizaje organizativo; 3) el intercambio de la información de manera fluida; 4) la educación de la fuerza de trabajo en la priorización de los recursos de información; 5) la eliminación de información innecesaria y redundante; 6) la eliminación de los silos de información; 7) la creación de datos críticos; 8) la entrada de datos por parte de los miembros de la organización (Cornella, 2000); 9) la localización fácil de grandes volúmenes de información, así como el intercambio de la información a través de una interfaz sencilla; 10) una mayor valoración de la información convirtiendo al usuario en proveedor de información (Sánchez Montero, 2000); y 11) la explotación de la información interna y externa - acceso, selección y difusión de información estructurada y no estructurada, interna y externa-.

4. Para la comunicación. La intranet permite y facilita: 1) la transferencia de información independientemente del contacto directo entre los miembros de una organización, siendo particularmente relevante en organizaciones de gran

Scire. $10: 2$ (jul.-dic. 2004) 25-39. 
tamaño, geográficamente dispersas, donde los miembros de la organización no se conocen entre ellos y donde la organización, como un todo, no sabe realmente lo que conoce (Stoddart, 2001); 2) la comunicación y el intercambio de información en el seno de la organización mediante la colaboración interdepartamental mejorando los flujos entre los miembros de la organización; 3) la comunicación y la interacción creando lo que algunos han llamado una knowledge connection, porque conecta las personas (Ruppel, 2001); y 4) la comunicación entre expertos para desarrollar y compartir nuevas especificaciones de productos y mejorar los resultados de nuevos productos, sobretodo en el ámbito empresarial (Scott, 1998).

5. Para la gestión del conocimiento: Permite la captura fácil de conocimiento no estructurado e implícito; aumenta la cultura informacional de los usuarios y se configura como la puerta de acceso al conocimiento de la empresa; incrementa el conocimiento y la capacidad de utilizar y compartir los recursos disponibles promoviendo una cultura organizativa común; facilita el conocimiento corporativo reduciendo las "islas independientes" y proporcionando una nueva forma de dibujar el conocimiento colectivo y la experiencia del personal, actual y pretérita (Fishenden, 1997); y posibilita tomas de decisiones rápidas y bien documentadas reduciendo costes.

\section{Factores de éxito}

1. De ámbito general. A nivel general para asegurarnos el éxito de una intranet debemos tener claras una serie de premisas (Pérez, 2002): a) crear un grupo especial de desarrollo, pues la intranet no es solo el trabajo de una persona, sino el resultado de un equipo proactivo, abierto y colaborativo; b) no identificar la intranet con el abandono de las formas tradicionales de la organización; c) canalizar necesidades e intereses de los usuarios y promover acciones para evitar reticencias y rechazos; y d) introducir el trabajo en red de manera progresiva en el entorno organizativo de trabajo.

2. En relación al diseño. Resulta de suma importancia el diseño del sistema de comunicación, ya que debe permitir la participación y un acceso fácil y rápido. El diseño y formato de los datos estratégicos es el primer paso en la transformación de los simples datos en conocimiento útil (Hoog, 1999). Destacamos los siguientes elementos clave de diseño: 1) auditoria — análisis de las necesidades informativas - ; 2) establecimiento de procesos de gestión y difusión de contenidos; 3 ) sistemas de recuperación de calidad; y 4) incorporación de elementos que ayuden a identificar conocimiento, por ejemplo, mapas de recursos, bases de datos de expertos y de best-practices. Lo más recomendable a la hora de diseñar una intranet sería empezar por un proyecto piloto de dimensiones manejables que pueda ser probado por un número limitado de usuarios y que permita al equipo

Scire. $10: 2$ (jul.-dic. 2004) 25-39. 
que lo desarrolle aprender de la experiencia: ¿qué contenidos interesan? ¿cómo estimular la participación? ¿qué modelo de gestión seguir? etc. (Cornella, 2000).

3. En relación con la estructura tecnológica. Por lo que concierne a la estructura tecnológica, la intranet debe incorporar los siguientes elementos (Eder, 2001): calidad de la información, desarrollo del sistema, calidad de la comunicación, herramientas de recuperación sofisticadas, y estructura y propósitos para continuar expandiendo y adaptando esta visión a lo largo del tiempo.

4. En relación con los usuarios. La existencia de usuarios activos es un factor clave. Si no se produce acción no puede existir intercambio o creación de conocimiento. También resulta importante que los usuarios actúen en función de sus propios intereses. La relación entre la información de la intranet y los miembros de la organización hace que esta sirva de base y les ayude a reflejar sus intereses, conocimientos y competencias. Por eso, resulta imprescindible definir una política de estimulación de los usuarios, ya que una intranet que nadie utiliza no tiene sentido. Debemos gestionar bien el contexto, entendido como la habilidad y la flexibilidad del sistema para permitir que los usuarios accedan a los contenidos en un entorno interactivo adecuado a cada individuo. Los cursos de formación y la demostración de cómo la intranet favorece el intercambio son maneras efectivas de promover el uso y el desarrollo de las intranets (Stoddart, 2001). Las intranets fomentan la cooperación, deben resultar prácticas para los usuarios finales, a los se debe escuchar y descubrir sus necesidades, analizando los log files y los patrones de comportamiento en la navegación. Por otro lado, también resulta útil dejar que los usuarios respondan e interactúen para determinar qué están utilizando y qué no (Fichter, 1999). Por último, hay que señalar que para crear una cultura de intercambio y mantenimiento de la información, el soporte de la dirección resulta esencial en las estrategias intranet.

5. En relación con la gestión del conocimiento. La clave fundamental consiste en gestionar la información con el objetivo que se convierta en conocimiento, es decir, que el conocimiento tácito que poseen las personas se difunda y se convierta en un factor fundamental de desarrollo. El intercambio de información debería convertirse en una práctica integrada en la cultura de la organización. La gestión del conocimiento debería integrarse en la planificación estratégica de la empresa, estableciendo objetivos concretos y asignando recursos. El éxito en la implantación de un sistema de gestión del conocimiento que aporte valor a la organización depende de la dirección general, de la cultura corporativa y del compromiso de los trabajadores con el proyecto. El conocimiento, el saber, implica depurar y añadir valor a la información, llegar a una solución o decisión en una situación real, interpretando estos dentro de un contexto. La transferencia de conocimientos, de best-practices y de know-how es lo que realmente aporta valor diferencial: reducción de costes, de tiempo y de toma de decisiones. Una

Scire. $10: 2$ (jul.-dic. 2004) 25-39. 
intranet efectiva debería permitir la captura de ideas a través de la estructuración de los datos y el almacenamiento y distribución del conocimiento, además de proveer una síntesis de la información clave para conseguir una promoción del intercambio de conocimiento. En esta línea, los mecanismos de retroalimentación resultan muy útiles.

6. Otros. Las intranets solo pueden tener éxito cuando toda la estrategia de la información está enfocada hacia los contenidos digitales. Una buena intranet necesita de tres elementos críticos: contenidos de calidad, buena tecnología y cultura positiva. Capturar y guardar estos tres elementos son el objetivo de una buena gestión de la intranet. Resulta de suma importancia determinar las prioridades. Primero, pensar en las personas y después en las tecnologías (Stoddart, 2001), ya que el éxito de las intranets depende de la sinergia de personas, contenidos y tecnología trabajando conjuntamente. Debemos mejorar la intranet mediante el aprendizaje y la innovación, invirtiendo tiempo evaluando la intranet de manera periódica. El intercambio de conocimiento depende no solo del uso particular de las tecnologías de la información sino del éxito en la creación de un entorno cooperativo de conocimiento con un enfoque hacia a la gestión del conocimiento (Pan, 2001).

\section{Limitaciones y retos}

La intranet se conforma como una parte del sistema de gestión de la información, pero no proporciona por sí sola ventajas competitivas, ya que no representa la única respuesta para solucionar los temas de conocimiento compartido. Las organizaciones deben utilizar además otras aproximaciones para integrar su conocimiento capital e intelectual. Pensar que cuando se ha creado una intranet ya se dispone de un sistema de gestión del conocimiento es un grave error, ya que nos encontramos sólo al principio del camino. Disponer de documentos en formato electrónico compartido resulta de entrada muy enriquecedor, pero no debemos olvidar que la mayoría del capital intelectual no se encuentra siempre en los documentos. Otro problema no resuelto es el de la información como poder, y depende de un esperado cambio cultural hacia el intercambio de la información. Las estructuras jerárquicas pueden limitar la información que se deposita en la intranet.

A continuación reseñamos cuatro de los errores más frecuentes a la hora de diseñar una intranet y que, por tanto, deberemos evitar (Curry, 2000): 1) faltan guías y políticas de uso de provisión de información en la intranet y una estructura tecnológica común para acceder a la información; 2) no se explica el desarrollo del contenido de la intranet a los usuarios limitando la posibilidad de añadirle valor; a menudo las decisiones quedan sólo en manos webmasters, cuando las intranets deben ser diseñadas en función de las necesidades de los usuarios con una planificación y objetivos claramente definidos a todos los niveles de la organi-

Scire. $10: 2$ (jul.-dic. 2004) 25-39. 
zación; 3) no existe una estrategia de comunicación, violando el principio de que las intranets deben asegurar que los mensajes enviados a los empleados permanecen luego fácilmente accesibles a todo el mundo; y 4) pensar que las intranets sólo sirven para crear una cultura del aprendizaje y del conocimiento, siendo que ofrecen también oportunidades para contribuir al proceso de mejora continua.

Los principales retos a los que deberemos hacer frente en la implementación de una intranet en una organización son siete. En primer lugar, es necesario saber distinguir entre información y conocimientos útiles para la organización, ya que estos son los que han de transferirse. En segundo lugar, procede determinar las herramientas a través de las cuales se difundirá y compartirá esta información. Existe el peligro de considerar la tecnología como una finalidad en sí misma y no como un medio de conocimiento, utilizando la intranet sólo para el almacenamiento de la información sin una estructuración y gestión eficaz de la misma. En tercer lugar, se debe dimensionar correctamente la importancia del factor humano. Debemos potenciar el elemento social y la comunicación humana en la transferencia de los conocimientos a través de un cambio o reestructuración de la cultura corporativa que potencie la motivación de los empleados y los ayude a entender la intranet como un entorno de cooperación. En cuarto lugar, es necesario determinar nuevos requerimientos para los usuarios. En este sentido debemos dedicar esfuerzos en potenciar habilidades en la gestión de contenidos informativos en entornos web, incrementar el dominio de las herramientas de búsqueda de Internet, facilitar nuevos conocimientos - HTML, XML, FTP...-, potenciar la formación de los trabajadores, y elaborar de nuevos productos para la gestión del conocimiento en Internet - mapas de recursos, bases de datos de expertos...-. En quinto lugar, hay que asumir que los beneficios de formar parte de una cultura informacional son difíciles de conseguir pero representan una contribución significativa en términos de ventajas competitivas. En sexto lugar, es necesario entender el hecho de que, aunque las intranets se presenten como herramientas que promueven la comunicación, colaboración y coordinación social, pueden producir efectos paradójicos. De manera irónica, generan fuerzas centrífugas que refuerzan la idea de que existen silos de conocimiento. La paradoja más grande es que resulten poco prácticas en contextos donde la gestión del conocimiento sea un objetivo clave (Newell, 1999, 2001). Algunos autores (Ali, 2001) son escépticos respecto la transferencia de conocimiento tácito en las intranets. A su entender, implementados de forma demasiado drástica, los mecanismos de intercambio y transferencia del conocimiento pueden destruir la creatividad de los miembros de la organización. Finalmente, en séptimo lugar, conviene considerar que la tecnología intranet tiende a ser de fácil implementación inicial, pero que se complica a partir de un tamaño que requiere el mantenimiento de grandes volúmenes de información y tipologías de red complejas.

Scire. $10: 2$ (jul.-dic. 2004) 25-39. 


\section{La necesidad de un cambio cultural}

El uso de intranets requiere de un cambio en la cultura corporativa por el que las organizaciones deben aprender a adaptarse a los cambios que exigen si quieren beneficiarse realmente de ellas. Utilizadas de manera incorrecta pueden intensificar la desconfianza, la desinformación y generar conflictos entre los trabajadores. Algunos las han bautizado como killer application de la gestión del conocimiento (Cohen, 1998).

Debemos concebir las intranets como un organismo vivo y evolutivo que se alimenta de información y conocimiento. Con una alimentación correcta - buena gestión, mantenimiento continuado e implementación bien planificada - pueden hacer crecer la red de conocimiento fomentando el aprendizaje, el intercambio y la colaboración. Las web-based-tecnologies crean una cultura cambiante y evolutiva, ya que conllevan implícito un lenguaje, una manera de pensar y un conjunto de valores y estándares. En palabras de Michael Vigue (senior vice-president of technology de la Boston-based Forum Corporation) (Cohem,1998)

Intranets are changing our expectations of quality and performance. They create a philosophy that says don't reinvent-take what you've got to a higher level. Do it faster, add more vale, and do it at a lower cost.

Aprendizaje y conocimiento no son simples funciones organizativas: tienen lugar en y entre personas individuales. Una organización solo puede "aprender" cuando los individuos son capaces de mostrar e intercambiar el conocimiento. Por tanto, el aprendizaje organizativo debe favorecer a los "aprendices individuales". Cuando un "aprendiz" potencial se enfrenta a una situación desconocida (conceptualmente caótica) pueden darse diferentes situaciones en las cuales puede aprender: 1) busca en las bases de conocimiento para ver si la situación se ha producido con anterioridad y la respuesta ya se conoce, esto es, aprende de la base de conocimiento organizativo); 2) encuentra respuestas relacionadas, pero no en las mismas circunstancias, y deriva una respuesta combinando piezas de conocimiento desde la base de conocimiento, creando nuevo conocimiento en el proceso; 3) genera nuevo conocimiento, normalmente creando acciones y anotando las respuestas, que es lo que, de manera organizada y sistemática, configura la investigación científica. El intercambio de conocimiento únicamente puede existir si la organización promueve una cultura en este sentido: las organizaciones estáticas y no colaborativas no estimulan el conocimiento, elemento estratégico en la gestión del conocimiento. En este sentido el aprendizaje es un requisito constante para asegurar el éxito de las intranets.

Evidenciamos pues, la necesidad de preparar un cambio de actitud en lo que concierne a los roles de la información y el conocimiento en la creación de nuevos productos y servicios. Necesitamos una cultura que enfatice una atmósfera que englobe trabajo en equipo (ethical culture), flexibilidad y innovación (develop-

Scire. $10: 2$ (jul.-dic. 2004) 25-39. 
mental culture) y políticas, procedimientos e innovación (hyerarchical culture) (Ruppel, 2001).

Por último, conviene tener en cuenta que, aunque la cultura influye en el uso de las intranets, el flujo es reversible: las intranets pueden causar cambios en la cultura existente; $y$, por tanto, necesitamos acciones de sensibilización encaminadas a entender que la información y el conocimiento aumentan el valor del aprendizaje del trabajo.

\section{Conclusiones}

Diseñar una intranet enfocada hacia la gestión del conocimiento implica la incorporación de aplicaciones que permitan el aprovechamiento de las ventajas que caracterizan a la tecnología web: openness, linking y networking. Además, la intranet debería utilizar sofisticadas aplicaciones que permitan navegar a través de la información no estructurada y encontrar los patrones entre las fuentes. Facilitando el acceso a las fuentes de comunicación, la intranet atraerá el interés de los usuarios.

El diseño de una intranet significa también considerar que la intranet no es un elemento aislado, sino que ha de relacionar todas las actividades que llevan a cabo todos los miembros de una organización. Una aplicación de intranet, como un motor de búsqueda, debería poder detectar si dos usuarios están interesados en un tema similar y utilizar esta metodología para incrementar la conciencia organizativa.

Las intranets representan una valiosa herramienta para las organizaciones. Para poder alcanzar este valor deben resolverse los conflictos que existen entre las percepciones que consideran la intranet como algo rápido, barato y estático, y la realidad de que, al igual que otros sistemas de información, debe planificarse y gestionarse adecuadamente.

Debemos introducir un sistema de calidad en el mantenimiento y desarrollo de las intranets. Un nivel óptimo de calidad y definición de las necesidades funcionales - actualizaciones, supervisión de enlaces, presentación y calidad de contenidos-, y desarrollar guías de la intranet, actualización de los procesos y una evaluación sistemática.

\section{Referencias}

Alavi, M.; Leidner, D. E. (2001). Knowledge Management and Knowledge Management Systems: Conceptual Foundations and Research Issues. // MIS Quarterly. 25:1 (2001) 107-136.

Ali, Y. (2001). The intranet and the management of making and using skills. // Journal of Knowledge Management. 5:1 (2001) 338-348.

Scire. $10: 2$ (jul.-dic. 2004) 25-39. 
Benett, R.; Gabriel, H. (1999). Organisation Factor and Knowledge Management within Large Marketing Departments: An Empirical Study. // Journal of Knowledge Management. 3:3 (1999) 212-224.

Chase, R. C. (1997). Effectively using intranets for knowledge management. // International Journal of Business Transformation. 1:1 (1997) 30-40.

Choo, C. W.; Detlor, B.; Turnbull, D. (2000). Web work: information seeking and knowledge work on the World Wide Web. Dordrecht: Kluwer Academic Publishers, 2000.

Cohen, S. (1998). Knowledge management's killer app: here's how an intranet can wire employees to information and knowledge without fragmentin a company's culture. // Training and Development. 52:1 (1998) 50-57.

Cornella, A. (2000). Infonomia!com. Bilbao: Deusto, 2000.

Curry, A.; Stancich, L. (2000). The Intranet: an intrinsic component of strategic information management?. // International Journal of Information Management. 20 (2000) 249-268.

Eder, L. B.; Igbaria, M. (2001). Determinants of intranets diffusion and infusion. // Omega: The international Journal of Management Science. 29 (2001) 233-242.

Fernandez de las Heras, José Manuel (2000). La intranet del conocimiento. // La Gestión del Conocimiento: retos y soluciones de los profesionales de la información: VII Jornadas Españolas de Documentación, Bilbao 19-20-21 octubre 2000. Bilbao: Universidad del País Vasco: Federación Española de Sociedades de Archivística, Biblioteconomía y Documentación, DL2000. 567-573.

Fichter, D. (1999). Seven habits for effective project managers // Online 23.4 (IلسلyAugust 1999). URL: 〈http://www.findarticles.com/cf_0/m1388/4_23/54891101 print.jhtml. > Consultado: 2002-05-23.

Fishenden, J. (1997). Managing intranets to improve business process.// Aslib Proceedings. 49:4 (April 1997) 90-96.

Hall, H. (2001). Input-friendliness: motivating knowledge sharing across intranets. // Journal of Information Science. 27:3 (2001) 139-146.

Hoog, Robert. (1999). Use your Intranet for Effective Knowledge. // E-Business Advisor. april 17:4. (april 1999). 27.

Institute for Information Studies (1993). The Knowledge economy: the nature of information in the 21st century. 1993-1994. // Annual Review. Institute for Information Studies, USA.

Intranets are not instant knowledge management systems (1998). // Information Outlook. 2:9 (September 1998). 42.

Mansell-Lewis, E. E. (1997). Intranet essentials. // Computer weekly. 9/10/97.

Newell, S.; Scarbrough, H. (1999). Intranets and knowledge management: complex process and ironic outcomes. // Proceedings of the Thirty-Second Annual Hawaii International Conference on System Sciences: Maui, Hawaii, January 5-8, 1999. Los Alamitos, CA, [etc.]: IEEE Computer Society Press, cop. 1999.

Newell, S.; Scarbrough, H. (2001). From global knowledge management to internal electronic fences: contradictory outcomes of intranet development. // British Journal of Managament. 12:2. (June 2001) 97-111.

Scire. $10: 2$ (jul.-dic. 2004) 25-39. 
Nonaka, I.; Konno, N. (1998). The concept of "Ba": building a foundation for knowledge creation. // California Management Review. 40:3 (1998) 40-55.

Nonaka, I.; Takeuchi, H. (1995). The knowledge-creating company. New York; Oxford: University Press, 1995.

Pan, S. L.; Hsieh, M.; Chen, H. (2001). Knowledge Sharing Throught Intranet-Based Learing: A Case Study Online Learning Center. // Journal of organizational computing and electronic comerse. 3:11 (2001) 179-195.

Perez, I. (2002). Intranet como herramienta de gestión del conocimiento. URL: <http: //www.rrhhmagazine.com/articulo/gescon/gescon7.asp>. Consultado: 14/6/2002.

Polanyi, A. (1966). The Tacit Dimension. London: Routledge and Kegan Paul, 1966.

Ruppel, C. P.; Harrington, S. J. (2001). Sharing knowledge throught intranets: a study of organizational culture and intranet implementation. // IEEE Transactions on Professional Communication. 44:1 (march 2001) 37.

Sanchez Montero, J. A. (2000). Intranets corporativas y gestión del conocimiento: el caso de una empresa del sector de la energía. // La Gestión del Conocimiento: retos y soluciones de los profesionales de la información: VII Jornadas Españolas de Documentación, Bilbao 19-20-21 octubre 2000. Bilbao: Universidad del País Vasco: Federación Española de Sociedades de Archivística, Biblioteconomía y Documentación, DL2000. 529-538.

Scott, J. E. (1998). Organizational knowledge and intranets. // Decision Suport Systems, 23 (1998) 3-17.

Standing, C.; Benson, S. (2000). Irradiating intranet knowledge: the role of the interface. // Journal of Knowledge Management, 4: 3 (2000) 244-251.

Stenmark, D. (2002a ). Designing the new intranet. (Tesis doctoral). Goteborg: Viktoria Institute, 2002.

Stenmark, D. (2002b). Information vs. knowledge: the role of intrantes in knowledge management. // Proceedings of the 35th Annual Hawaii International Conference on System Sciences: 7-10 January, 2002, Big Island, Hawaii / edited by Ralph H. Sprague, Jr. Los Alamitos, CA, [etc.]: IEEE Computer Society Press, c2002.

Stoddart, L. (2001). Managing intranets to encourage knowledge sharing: opportunities and constraints. // Online Information Review. 25:1 (2001) 19-28.

Tellen, S. L. (1996). Intranet organization: stategies for managing change. // Amdahl Corporation. URL:<http://www.iorg.com/intranetorg/index.html> . Consultado: $22 / 7 / 2002$. 